\title{
FACETTEN DER JOSEPHINE BAKER
}

\section{Christa Bruckner-Haring und Ildikó Keikutt-Licht}

"Sie ist die echteste. In ihr ist das Negertum am reinsten. Sie gibt den Rhythmus. Kommt aus dem Blute. Aus dem Urwald."So schreibt die Berliner Börsen-Zeitung am 7. Januar 1926 über Josephine Baker als den Star der Show La Revue nègre. Baker eroberte während ihres kurzen Gastspiels im Nelson Theater Berlin in ebensolchem Sturm wie sie auch Paris überwältigt hatte. Sie war die wilde Urwaldschönheit, die schwarze Venus, schlechthin die Verkörperung kolonialer Exotik-Phantasien. Diese entzündeten sich lodernd an den aus Amerika importierten, Mitte der 1920er Jahre auf den Bühnen von Paris populär gemachten Revues nègres, obgleich die Faszination des Exotischen, das Begehren des Fremden nicht neu war. Die bildende Kunst, die Literatur, das Theater und der klassische Bühnentanz entwickelten bereits seit der Jahrhundertwende ihr Interesse am Primitiven als Inspirationsquelle. Davon zeugen Picassos Les Demoiselles d'Avignon aus dem Jahr 1907, genauso wie Diaghilevs Ballets Russes, mit denen er Choreographien wie Cléopâtre (1909) und Shéhérazade (1910) in exotischen Settings schuf.

Baker hatte ihre Reise nach Paris angetreten als eine Reise mit ungewissem Ausgang, was den Erfolg der Show und ihrer eigenen Karriere anging. Sie war in den USA im Musical Shuffle Along von Eubie Blake und Noble Sissle als »comedy chorus girl « aufgefallen und hatte sich damit für weitere Engagements empfohlen. Ein »comedy chorus girl« war üblicherweise das Mädchen am Ende der Reihe von Tänzerinnen, das zunächst durch seine Unbeholfenheit das Publikum zum Lachen brachte und schließlich alle anderen Tänzerinnen überflügelte. Beide Qualitäten legten den Grundstein für Bakers weitere Laufbahn: Groteske, parodistische Züge kombinierten sich in ihren Auftritten mit einer besonderen tänzerischen Begabung. Beides alleine hätte jedoch nicht gereicht, um die Ikone zu schaffen, zu der sie - beginnend mit ihrem ersten Erscheinen im Théâtre des Champs-Élysée - aufstieg.

Bakers enorme Popularität mit ihrer publikumswirksamen Inszenierung als nackte, exotische Schönheit zu erklären, scheint naheliegend, wird dem 
Phänomen Baker, das seine Faszination durch jegliche Gesellschaftsschichten hindurch entfalten konnte, jedoch auch nur auf den ersten Blick gerecht. Die Figur der Baker hat mehr zu bieten: Sie ist facettenreich. Wie aber war ein solcher Facettenreichtum bei einem so scheinbar plakativschlichten Image möglich?

Entscheidend für ihren Erfolg war die erste Phase ihrer Karriere von 1925 bis 1935, in der sie ihr grundlegendes Image schuf, das im Folgenden mit dem Fokus auf den verschiedenen Facetten der Ausdrucksweisen »Tanz « und »Gesang « beleuchtet werden soll.

\section{Die $\gg$ Ur-Baker «}

Dreh- und Angelpunkt des Bildes der »Ur-Baker « ist der von ihr in der Revue nègre erstmals aufgeführte »danse de sauvage«, der »Tanz der Wilden«. Ihr Auftritt im Bananenrock auf der Bühne der Folies Bergère stellte das entscheidende Moment für ihre Popularität dar und zeichnete ein Bild, mit dem sie für immer verbunden blieb. Die Kostümierung aus Plüschbananen und ihre Nacktheit waren entscheidende Details der »Ur-Baker«. Die rhythmischen, isolierten Bewegungen ihres scheinbar knochenlosen Körpers, gepaart mit grotesken Posen, bedienten die stereotype, sexualisierte Phantasie der erotischen Urwaldschönheit.

Die Figur mit Namen Fatou, die Bakers »danse de sauvage« zugrunde liegt, entstammte Pierre Lotis Roman Le Roman d'un spahi (1881). Sie belegt die Stereotypien speisende Rückbindung der dunkelhäutigen Frau an den Kontinent Afrika, indem sie »allein über die Hautfarbe, die sich bis in die seelischen Tiefen des >coeur noir < fortsetzt, [...] unwiederbringlich und unauslöschbar an ihre afrikanische Heimaterde gebunden « (Hölz 2002: 178) ist. Die Inszenierung der »Ur-Baker« fußt damit auf der Perspektive des kolonialen Blicks und der damit einhergehenden Ambivalenz zwischen Beherrschen und Begehren, getragen durch den Glauben des weißen Europas an die eigene technische wie moralische Überlegenheit und demgegenüber die Sehnsucht nach Ursprünglichkeit. Monika Ehlers, Eva Lezzi und Sandra Schramm heben in Bezug auf das »colonial desire « die Geschlechterrollen hervor, indem sie formulieren:

»Die im Hinblick auf die Kolonien verhandelten sexuellen und rassischen Stereotypen sind verbunden mit dem Versuch einer klaren Zuordnung von Geschlechterpositionen. So wird [...] die fremde Kultur häufig mit Weiblichkeit und schrankenloser Sexualität assoziiert, die eigene hingegen mit Männlichkeit und überlegener Rationalität« (Ehlers/Lezzi 2003: 2). 
Die Frau als sexualisiertes Objekt. Baker als das Objekt der Begierde. Eine performativ von außen zugeschriebene Rolle, wie der von Judith Butler in ihren Gender Studies entwickelte Performativitätsbegriff belegt. Butler geht davon aus, dass Subjekten ihr Verhalten und Sein nicht eingeschrieben ist, sondern dieses erst durch diskursives Auferlegen bestimmt wird:

»Acts, gestures and desire produce the effect of an internalized core or substance, but produce this on the surface of the body [...]. Such acts, gestures, enactments, generally construed, are performative in the sense that the essence or identity that they otherwise purport to express are fabrications manufactured and sustained through corporeal signs and other discursive means« (Butler 1990: 136).

\section{Der Bananenrock}

Das Aufzeigen stereotyper Exotik-Phantasien kann jedoch lediglich ein Ausgangspunkt sein. Wie nun ging Baker mit diesen Stereotypen um? Anhand des »banana dance « lassen sich zwei wesentliche Merkmale in den Blick nehmen: ihre Kostümierung und ihr Tanzen. Fällt der Blick zunächst auf ihre Kostümierung, stellt sich eine Frage, mit der sich auch Anne Anlin Cheng in ihrem Buch Second Skin intensiv auseinandersetzt: ob Baker letztlich als angezogen oder ausgezogen, als nackt oder kostümiert zu beschreiben ist.

Stellt man ihre Nacktheit in den Kontext der Inszenierung von Blöße in den 1920er Jahren, entpuppt sich diese als ein Oberflächenphänomen, als eine entsubjektivierte Hülle. So sind die Revuetheater ein Ort der ZurSchau-Stellung der Frau und ihrer Nacktheit und Spiegel einer Zeit, die, in den Worten des Literaten und Zeitzeugen Felix Salten (1924: 3), »den Frauenkörper ohne Verschleierung als öffentliches Schaustück« annimmt. Die nackte Haut ist die Oberfläche, die plakativ-sexualisiert geschlechtliche wie Rassen-Differenzen darbietet.

Baker ziert sich als Hülle mit der Nichtigkeit eines Obst-Röckchens, das als ungewöhnliches Accessoire besticht. Durch die Vielzahl von möglichen Perspektiven der Deutung und Betrachtung dieser Kostümierung eröffnet sich ein Verwirrspiel, bei dem die Rollenverteilung von Betrachter und zu betrachtendem Objekt durchkreuzt wird. Nur einige dieser Deutungen seien mit den folgenden, von Cheng pointiert herausgearbeiteten Fragen umrissen: Sind die Bananen an Bakers Hüfte eine phallische oder Rassen bezogene Anspielung? Bezieht sich das Begehren des Publikums auf die Bananen oder den Körper, den sie »bedecken«? Ist die Banane auf koloniale Ökonomie zu 
beziehen? Geht es um Männlichkeit oder Weiblichkeit? (vgl. Cheng 2011: 44f.) Das Bananenröckchen dient weitaus weniger der sexualisierten Stereotypisierung als ihrer Dekonstruktion. Bakers Körper erhält durch die Bananen Macht über all die Männlichkeit, drückt aber gleichzeitig auch das Männliche, in heterosexueller wie homoerotischer Sicht aus. Baker erscheint geschlechtlich indifferent, sie wird zum Bezugspunkt für das männliche wie weibliche weiße Publikum. Die Grenze zwischen Begehren und Identifikation verschwimmt. Der Betrachter wird nicht vor dem fremden Anderen bewahrt, sondern in ein Szenario hineingezogen, indem er selbst die Züge dieses Anderen überstreift (vgl. ebd.: 47).

\section{Bakers Tanz}

Eine entscheidende Komponente dieses Images ist der Tanz, der erstaunlicherweise auch bei Cheng weitgehend unbeachtet bleibt. Die Bananenrock-Inszenierung wird vor allem deshalb zum entscheidenden Image Bakers, weil sie durch den dazugehörigen Tanz als grundlegend gefestigt wird. Bakers Bewegungsmaterial fußt auf Tanzschritten und Elementen, die im Kontext der afroamerikanischen Bevölkerung entstanden, im Umfeld der Minstrel- und Vaudeville-Shows variiert und teilweise in gemäßigter Form als Gesellschaftstänze populär gemacht worden waren. Die Bewegungssprache wurzelte in traditionellen afrikanischen Tänzen. Die Charakteristika des afrikanischen Tanzes sind Bewegungselemente, die sich auch in Bakers Tanzstil finden: die gebeugte Körperhaltung, gleitende, schleifende Schritte, von der Körpermitte, dem Becken aus gesteuerte, einzeln isolierte Bewegungen, Nachahmungen von Tieren, der pulsierende Rhythmus und das flexible, improvisierende Agieren (vgl. Levine 1977: 16). Ferner wurden der Charleston und dessen markantesten Merkmale, der zitternde Körper, die wechselnden $\mathrm{X}$ - und O-Beine sowie die nach Innen und Außen drehenden Füße, mit Baker assoziiert.

In besonderer Weise auffällig in Bakers Performance ist die Kombination von Tanzelementen mit parodistischen Gesten. Dazu gehören das aus der Minstrel-Tradition entlehnte Verdrehen der Augen, ihr »monkey walk«, also das Nachahmen eines Affen, das Imitieren des Kopfnickens eines Huhns sowie Herausstrecken des Pos als Abschluss ihres Tanzens.

Parodie war im Zusammenhang künstlerischer afroamerikanischer Ausdrucksweisen nicht neu. Das Parodieren von Afroamerikanern durch weiße Schauspieler im Minstrel-Theater mit dem markanten Merkmal des »black face« wurde durch afroamerikanische Entertainer übernommen. Die Para- 
doxie des doppelten »black face « und des Parodierens der eigenen Bevölkerung ermöglichte das Ausbilden einer Doppelbödigkeit, die durch das groteske Überzeichnen rassistischer Stereotypien diese ad absurdum führte. Über die Minstreltradition hinaus haben die Studien von Henry Louis Gates (1984) diese Doppelbödigkeit als besonderes Merkmal eines künstlerischen, afroamerikanischen Ausdrucks offengelegt. So ist der Cakewalk wiederum ein frühes Beispiel aus der afroamerikanischen Tanzgeschichte, der hinter der Maske des geselligen Tanzens die Tanzformen der euroamerikanischen Oberschicht parodierte. Bakers ins Extreme gekehrte Inszenierung des Primitiven lässt sich somit als Maskerade begreifen. Michael Borshuk bemerkt dahingehend: »Baker's parodic dances exposed the error of the converse assumption in the schema: that blacks were inherently inferior primitives « (Borshuk 2001: 50).

In der Betrachtung Bakers ist es jedoch wichtig, noch weiter über diesen Ansatz hinaus zu gehen, um die Aspekte parodistischer Überzeichnung und ihrer Inszenierung als entsubjektivierte Hülle zu verdeutlichen und einzuordnen. Erhellend ist hier Bakers Begegnung mit dem Theaterregisseur Max Reinhardt im Zuge des Gastspiels der Revue nègre in Berlin. Reinhardt versuchte sie zu einer seriösen Bühnenlaufbahn zu bewegen. „Mit dieser Körperbeherrschung, diesen pantomimischen Fähigkeiten, könnte ich«, so formuliert Reinhardt, »das Gefühl in einer Weise wiedergeben, in der es noch nie dargestellt worden ist « (zit. n. Gumbrecht 2003: 253). In ihrem Ausdruck sieht er also den Verweis auf etwas Substanzielles, vielleicht Ursprüngliches. Auf der anderen Seite, dies geht aus den Tagebüchern seines Freundes Harry Graf Kessler hervor, erlebten sie Baker als ein »reines Oberflächenphänomen«. Kessler schreibt:

»Die Baker tanzte mit äußerster Groteskkunst und Stilreinheit, wie eine [...] archaische Figur, die Akrobatik treibt, ohne je aus ihrem Stil herauszufallen. Sie tut das stundenlang scheinbar ohne Ermüdung [...]. Sie wird dabei nicht einmal warm, sondern behält eine frische, kühle, trockene Haut. Ein bezauberndes Wesen, aber fast ganz unerotisch« (Kessler, zit. n. Gumbrecht 2003: 254).

Eben in dieser Ambivalenz, in der Unsicherheit des Betrachters über die Bedeutung ihres Tanzes und in dessen Hoffnung, dahinter das begehrte Wahre zu finden, inszeniert sich Baker.

Auf der einen Seite nutzt sie - wie Gabriele Klein für die Kunstform »Tanz« herausgearbeitet hat - »die soziale Wirksamkeit des Tanzes«, die »in seiner Performanz « liegt: »Im Akt des Tanzens liegt das widerständige Potenzial des Tanzes, nämlich, herkömmliche Ordnungen zu unterlaufen 
und andere Wahrnehmungen und Erfahrungen möglich zu machen« (Klein 2010: 142). Auf der anderen Seite spielt Baker mit den Symbolen kolonialer Phantasien und dem Begehren ihrer Betrachter. Die Mehrdeutigkeit ihrer »Oberfläche« ist zentral. Damit lässt sich auch ihre Absage an Reinhardt und das »seriöse « Theater erklären. Sie brauchte das Umfeld der Music-Hall als Ort kolonialer Phantasien, in dem sie die Ambivalenz ihres Tanzens ausspielen konnte.

\section{Das Bananenrock-Image}

Baker verkleidete die Oberfläche ihres Körpers über das Tanzen als performativem Akt mit dem Bananenrock-Image. Dieses grundlegende Image festigte sie in Wiederholungen des »banana dance « in der ganzen Welt. Vor dem Hintergrund dieser grundlegenden Inszenierung - und hier kann im Gegensatz zu Bennetta Jules-Rosette (2007) für eine Hierarchisierung der Images plädiert werden - gewinnen alle anderen ihrer Image-Inszenierungen ihre besondere Wirkung. Das Bananenröckchen, wenn auch nur imaginär, bleibt die als Vergleich dienende Basis. Ihre weiteren Maskeraden trugen ein Übriges dazu bei, Bakers Publikum in der Ungewissheit über die Gültigkeit akzeptierter Stereotypisierungen zu belassen (vgl. Borshuk 2001: 55). So auch ihr Gesang, der sich vom Bild des Primitiven entfernt.

\section{Der Gesang als Image-Facette}

Die Gesangsstilistik Bakers, die anhand ausgewählter Beispiele und selbst angefertigter Transkriptionen vergegenwärtigt werden soll, stellt einen besonders eindrücklichen Teil ihres Images dar.

Insbesondere seit dem Beginn der 1930er Jahre wurde Bakers Gesang neben dem Tanz zu einem wichtigen Element und einem weiteren bedeutenden Ausdrucksmittel in ihren Aufführungen. Ohne jegliche Gesangsausbildung konnte sie sich erfolgreich als Sängerin präsentieren und nahm im Laufe ihrer Karriere rund 140 Stücke auf, hauptsächlich US-amerikanische Jazzstandards aus den 1920er und 1930er Jahren, darunter »| Found A New Baby« (1926, Musik: Spencer Williams / Text: Jack Palmer), »Pretty Little Baby« (1926, Musik: Ben Bernie, Phil Baker / Text: Sid Silvers) oder »Bye Bye Blackbird« (1926, Musik: Ray Henderson / Text: Mort Dixon), sowie Chansons, beispielsweise »J'ai deux amours« (1930, Musik: Vincent Scotto / Text: Géo Koger, Henri Varna), »Ram-Pam-Pam« (1930, Musik: Alfredo de 
Vita / Text: Jean Tranchant) oder »Haïti« (1934, Musik: Vincent Scotto / Text: Géo Koger ).

\section{Want To Yodel}

»I Want To Yodel « aus dem Jahr 1926 ist eines der frühesten aufgenommenen Lieder von Baker. ${ }^{1}$ Das Stück wurde komponiert von dem aus New Orleans stammenden Jazzpianisten und Komponisten Spencer Williams (1889-1965), der 1925 für drei Jahre nach Paris reiste und dort für die Show Revue négre und auch Baker komponierte. Die Tonart des Stücks ist Bb-Dur, und die Taktart ist 4/4. In formaler Hinsicht (siehe Tabelle 1) folgt nach einer kurzen instrumentalen Introduktion mit einem harmonischen Turnaround $\left(\mathrm{Bb}-\mathrm{CHdim}{ }^{7}-\mathrm{Cm}^{7}-\mathrm{F}^{7}\right)$ Teil $\mathrm{A}$, eine 16-taktige gesungene Strophe. Anschließend folgt Teil B, ein Refrain, in dessen Wiederholung B2 Baker durchwegs auf improvisatorische Art über das bestehende Form- und Harmonieschema jodelt. Auf musikalisch ähnliche Weise endet das Stück in einer kurzen Coda.

\begin{tabular}{|l|l|l|l|l|l|}
\hline Formteil & Introduktion & A & B1 & B2 & Coda \\
\hline Takte & $4(2+2)$ & 16 & $18(8+8+2)$ & $18(8+8+2)$ & 4 \\
\hline & $\begin{array}{l}\text { 2-taktiger } \\
\text { Turnaround } \\
\text { I-\#IIdim }-1 I \mathrm{I}^{7}-\mathrm{V}^{7}\end{array}$ & $\begin{array}{l}\text { begleiteter } \\
\text { Gesang }\end{array}$ & $\begin{array}{l}\text { gesungen } \\
\text { mit Fill-Ins } \\
(» J o d e l n \ll)\end{array}$ & $\begin{array}{l}\text { Gesangs- } \\
\text { improvisation } \\
(» J o d e l n \ll)\end{array}$ & $\begin{array}{l}\text { Gesangs- } \\
\text { improvisation } \\
(» \text { Jodeln«) }\end{array}$ \\
\hline Tempo & $J=102$ & & $J=106$ & & \\
\hline
\end{tabular}

Tabelle 1: »I Want To Yodel«, Formablauf

Die Stilistik dieses Stücks ist dem Dixieland Jazz, einer Form des Oldtime Jazz, zuzuordnen. In der für diesen Stil typischen Besetzung mit der Trompete als Lead-Instrument wird basierend auf einer ternären Grundlage musiziert. Hinzu kommt Bakers Gesang mit den Jodel-Passagen, die ohne Text auf Lautsilben gesungen werden. Das Einbinden von Jodlern in die JazzStilistik war zu jener Zeit keine Neuheit: Aus der Verbindung von Elementen des Blues, des Oldtime Jazz und dem charakteristischem Jodeln aus alpenländischen Traditionen entstand der Musikstil »Blue Yodeling «, in den USA ein populärer Stil während der 1920er und 1930er Jahre, der bei CountrySongs sowie in Minstrel- und Vaudeville-Shows verwendet wurde. ${ }^{2}$

1 Vgl. Baker 1999: Track 7.

2 »Blue Yodeling « wurde zu einem Markenzeichen von Jimmie Rodgers (18971933), der in seinen Liedern in Country-Stilistik jodelte. Auch in Kanada und Australien war »Blue Yodeling « ein populärer Musikstil (vgl. Herzhaft 1992: 364ff.). 

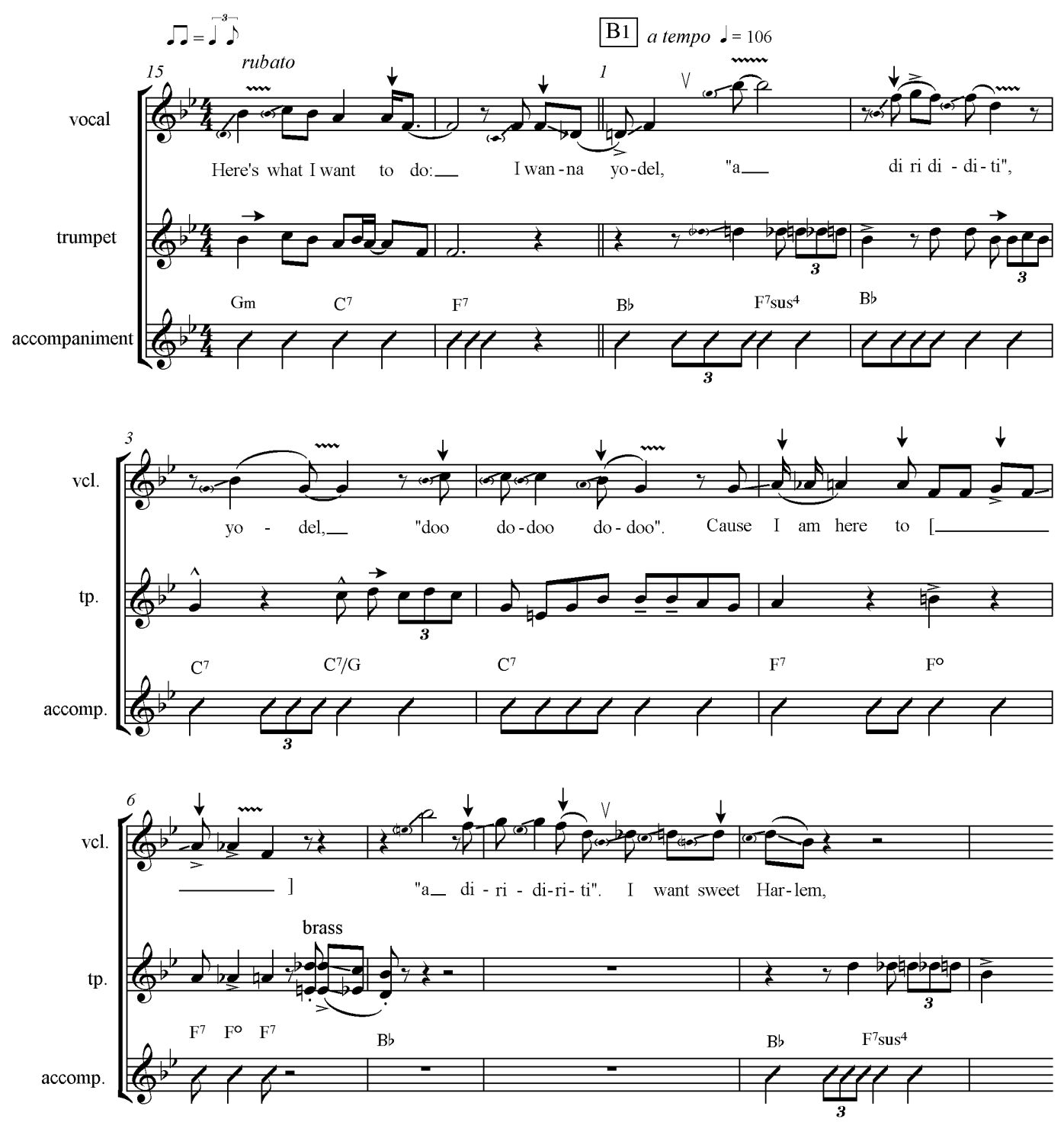

Beispiel 1: »I Want To Yodel«, Überleitung und B1 (T. 1-9) ${ }^{3}$

Musikalisch gesehen werden durch das Jodeln Bakers gesangliche Stärken, ihr Sopran und ihre helle Stimmfarbe, hervorgehoben. Ihre Stimme ist bereits außergewöhnlich ausgeglichen, auch wenn Baker in der tieferen Lage (wie in Beispiel 1 in der ersten Oktave) noch weniger Klangvolumen und Sonorität aufweisen kann. In ihrer Melodiegestaltung bleibt Baker einfach: Sie verwendet hauptsächlich Akkordtöne und bewegt sich überwiegend in kleinen Tonfortschreitungen, meist innerhalb eines engen Tonraums. Während der Jodel-Passagen ab der zweiten Oktavlage werden auffallend viele Portamenti in ihre Melodielinien eingebaut. In rhythmischer Hinsicht gebraucht

3 Erläuterung der Sonderzeichen: Linie zwischen Notenköpfen = Portamento; $\rightarrow$ $=$ früher als notiert; $\leftarrow=$ später als notiert; $\uparrow=$ höher als notiert, $\downarrow=$ tiefer als notiert. 
Baker wie im Beispiel 1 vielfach Off-Beat-Akzentuierungen und beginnt auch ihre Gesangslinien häufig auf dem Off-Beat. Diese Rhythmisierung taucht während des gesamten Stückes immer wieder auf.

Die Trompete spielt im Teil B in für den Blues typischer Call-andResponse-Manier Fill-Ins beziehungsweise improvisiert im Hintergrund in antiphonischer Struktur und gemeinsam mit Baker während ihrer Jodel-Passagen, teilweise als Parallelmelodie. Dabei spielt die Trompete ohne allzu viele Portamenti und die für die Stilistik typische Hot-Intonation.

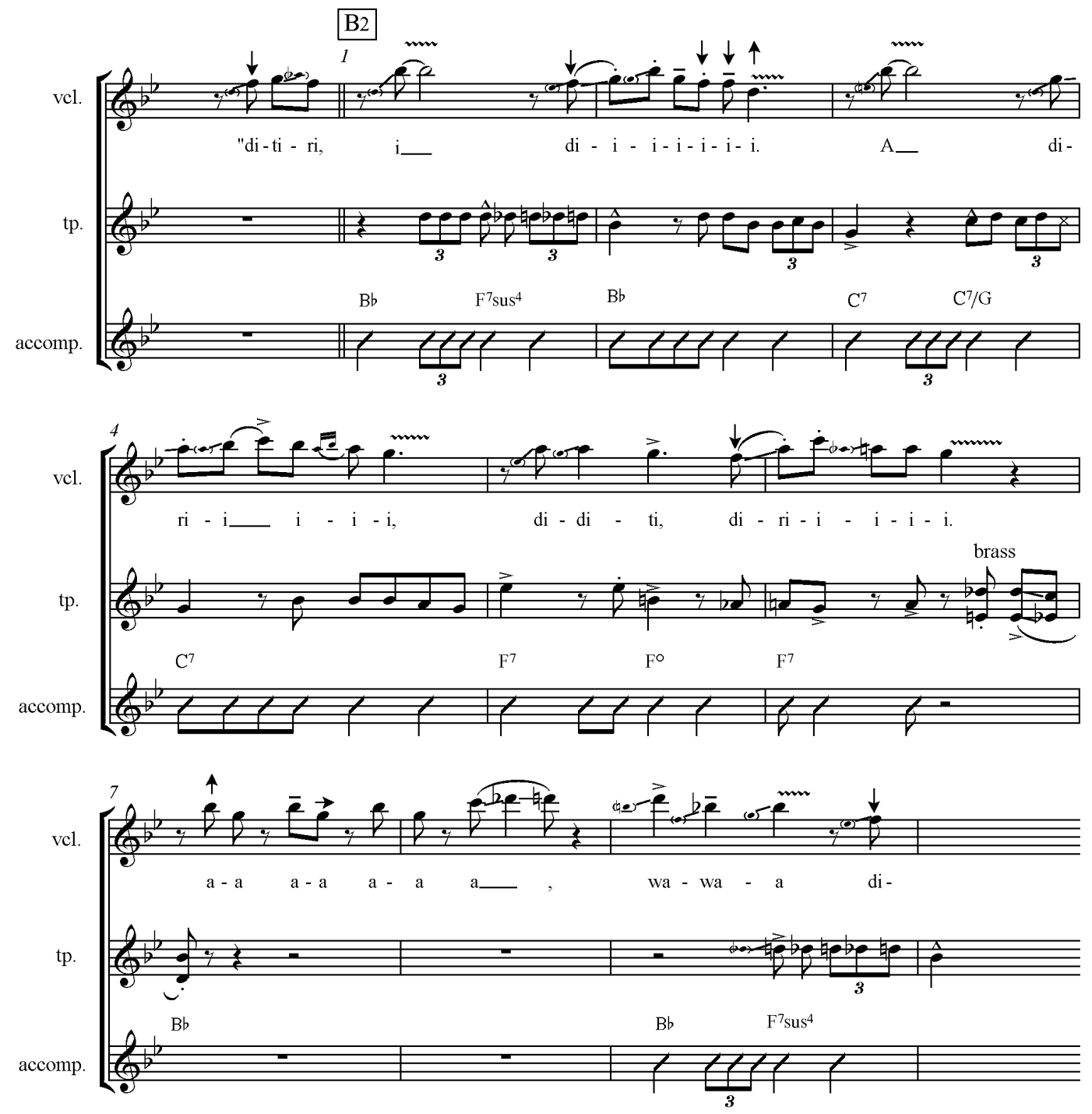

Beispiel 2: »I Want To Yodel«, B2 (T. 1-9)

Im zweiten Refrain singt Baker in improvisatorischer Art innerhalb eines höheren Tonraums im Bereich zwischen $f^{2}$ und $d^{3}$. Dabei verwendet sie teilweise ein Vibrato, meist über länger gehaltene Noten, beispielsweise während des zentralen Tons $b^{2}$. Wie bereits im Teil B1 sind häufig Blue Notes (kleine Terz und kleine Septim) im Gesang und in der Trompete zu hören. 
Als Kontrast zu den »unsauberen« Blue Notes singt Baker in diesem Teil die kleine Terz vom $b^{2}$ abwärts drei Mal hintereinander in heller Stimmfarbe und sauberer Intonation (T.7-8), vermutlich eine Kuckucks-Imitation. Zusätzlich wird das Motiv durch ein Break sowie durch die synkopische Rhythmisierung und die dadurch entstehende rhythmische Verschiebung hervorgehoben. Danach folgt sogleich ein ausgedehnter und kontrastierender Schleifer nach oben bis zum melodischen Höhepunkt des gesamten Stückes.

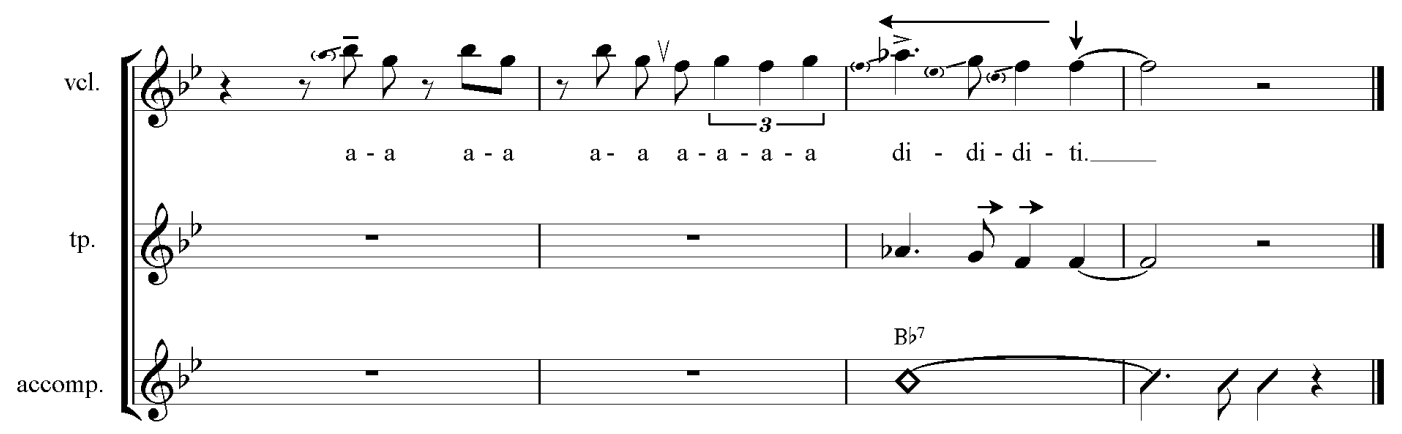

Beispiel 3: »I Want To Yodel«, Coda

Am Ende des Stücks erscheint das Kuckucksmotiv in der Coda erneut; wiederum kontrastiert durch die Weiterführung der Phrase, wo Baker zunächst die Blue Note $a s^{2}$, also die kleine Septime der Tonika $\left(\mathrm{Bb}^{7}\right)$ betont. Schließlich erfolgt die in musikalischer Hinsicht weniger spektakuläre Auflösung in die reine Quint.

\section{J'ai deux amours}

Das im Jahr 1930 speziell für Baker geschriebene Stück »J'ai deux amours« zählt zu ihren bekanntesten Liedern und entwickelte sich zu ihrer persönlichen Hymne. Der Text stammt von den französischen Autoren Géo Koger (1894-1975) und Henri Varna (1887-1969), die Musik wurde vom französischen Komponisten Vincent Scotto (1874-1952) komponiert. Eine frühe bekannt gewordene Version stammt aus dem Jahr 1931, aufgenommen in Paris gemeinsam mit dem französischen Sänger Adrien Lamy. ${ }^{4}$ Die Tonart des Stücks ist F-Dur, die Taktart 4/4, und die Form ist eine zweiteilige Liedform A-B mit Wiederholung (siehe Tabelle 2). Nach der instrumental ausgeführten Introduktion folgen die Strophe A und der in sich dreigliedrige Refrain B, wobei die jeweils ersten und letzten acht Takte harmonisch und melodisch fast identisch sind. Der zweite Refrain wird von Lamy und Baker gesungen.

4 Vgl. Baker 1968: Side 1, Track 1. 


\begin{tabular}{|c|c|c|c|c|c|}
\hline Formteil & Introduktion & $\mathrm{A} 1$ & B1 & $A 2$ & B2 \\
\hline \multirow[t]{2}{*}{ Takte } & 4 & $9(8+1)$ & $24(8+8+8)$ & $9(8+1)$ & $24(8+8+8)$ \\
\hline & instrumental & $\begin{array}{l}\text { Strophe: } \\
\text { gesungen } \\
\text { von Baker }\end{array}$ & $\begin{array}{l}\text { Refrain: } \\
\text { gesungen } \\
\text { von Baker }\end{array}$ & $\begin{array}{l}\text { Strophe: } \\
\text { gesungen } \\
\text { von Baker }\end{array}$ & $\begin{array}{l}\text { Refrain: } \\
\text { gesungen von } \\
\text { Lamy mit Fill-Ins } \\
\text { von Baker bzw. } \\
\text { Baker solistisch }\end{array}$ \\
\hline Tempo & $d=118$ & $J=$ ca. 76 & $d=109$ & $d=$ ca. 76 & $J=109$ \\
\hline
\end{tabular}

Tabelle 2: »J'ai deux amours«, Formablauf

Dieses Stück kann in stilistischer Hinsicht dem Genre Chanson zugeordnet werden. Dabei liegt eine große Bedeutung in der Textaussage, die in diesem Fall einen melancholischen Charakter hat: Es geht um die Unsicherheit, nicht genau zu wissen, wo man hingehört, verbunden mit gleichzeitigem Fern- und Heimweh sowie einer gewissen Sehnsucht.

\section{»J'ai deux amours $\aleph^{5}$}

A (1. Strophe)

On dit qu'au-delà des mers,

Man sagt, dass jenseits der Meere,

Là-bas sous le ciel clair,

Il existe une cité, au séjour enchanté. dort unter dem klaren Himmel,

Et sous les grands arbres noirs,

Chaque soir,

Vers elle s'en va tout mon espoir. eine Stadt existiert, wo man verzaubert wird. Und unter den großen schwarzen Bäumen, jeden Abend, geht ihr meine ganze Hoffnung entgegen.

B (Refrain)

J'ai deux amours

Ich habe zwei Lieben,

Mon pays et Paris. mein Land und Paris.

Par eux toujours, Bei ihnen für immer

Mon coeur est ravi. ist mein Herz entzückt.

Manhattan est belle, Manhattan ist wunderschön,

Mais à quoi bon le nier: aber wem nützt es zu leugnen:

Ce qui m'ensorcelle Das, was mich bezaubert, C'est Paris, Paris tout entier. ist Paris, ganz Paris.

Le voir un jour Es eines Tages zu sehen,

C'est mon rêve joli. ist mein schöner Traum.

J'ai deux amours Ich habe zwei Lieben, Mon pays et Paris. mein Land und Paris.

5 Deutsche Übersetzung von den Autorinnen. 
A (2. Strophe)

Quand sur la rive parfois

Au lointain j'aperçois

Un paquebot qui s'en va,

Vers lui je tends les bras

Et le cœur battant d'émoi.

À mi-voix

Doucement je dis »emporte-moi!«
Wenn manchmal am Ufer

ich in der Ferne sehe

ein Passagierschiff, das dahinfährt, strecke ich ihm meine Hände entgegen, und mein Herz, das schlägt vor Aufregung.

Halblaut

sage ich sanft »Nimm mich mit!«

Inhaltlich wurde dieser Text mit Bakers Leben in Verbindung gebracht, da daraus jene Ambivalenz herausgefiltert wurde, die für sie angeblich zutreffend war: das Hin- und Hergerissensein zwischen zwei für sie wichtigen Orten, nämlich ihrer Heimat USA und ihrer Wahlheimat Frankreich: »Zwei Lieben habe ich, mein Land und Paris«.

In musikalischer Hinsicht präsentiert sich Baker im Gegensatz zu ihren Tanz-Inszenierungen in einem stark kontrastierenden Setting: Ihr Gesangsstil ist dem populären französischen Chanson-Stil angepasst, auf den auch die Jazzmusik und speziell der Swing damals einen nicht unwesentlichen Einfluss hatten, was sich unter anderem auf die Art der Begleitung und die Beat-Unterteilung sowie die Harmonien auswirkte.

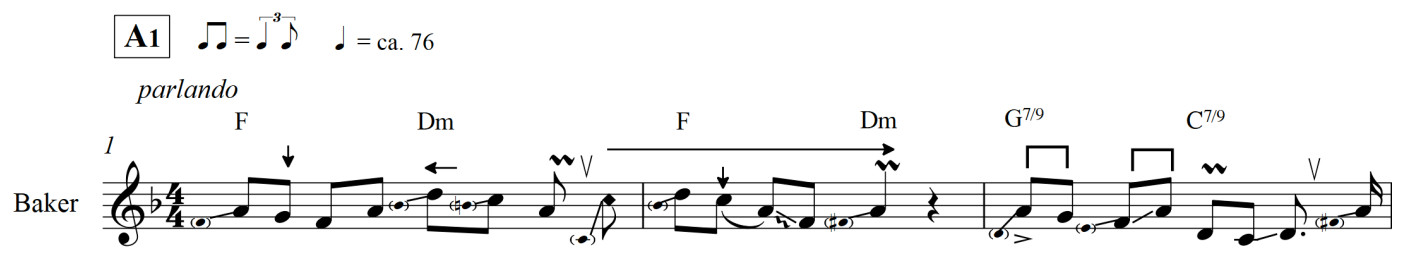

On dit qu'au-de - là des mers, là - bassous le ciel clair,

il ex -iste u-ne ci-té, au

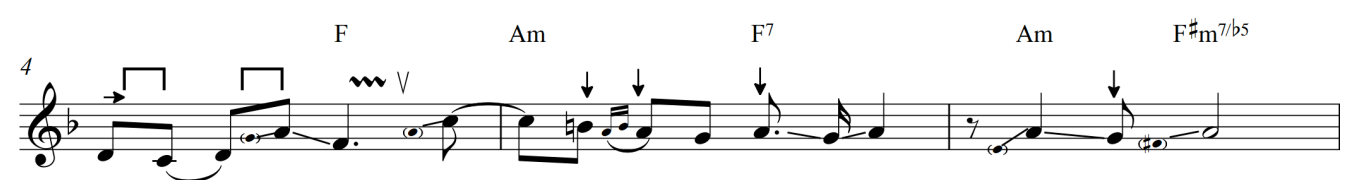

sé - jour en - chan - té.

Et_

sous les grands ar - bres noirs,

cha - que soir,

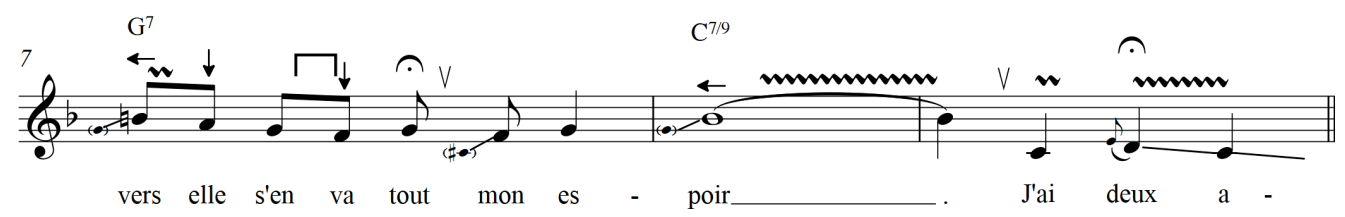

Beispiel 4: »J'ai deux amours«, $A 1^{6}$

6 Erläuterung der Sonderzeichen: rautenförmiger Notenkopf = stark gesprochene Töne; $\sqcap$ = gerade Achtelnoten. 
Baker verwendet in ihrer Interpretation, insbesondere für die Gestaltung der Strophe im Tonraum der ersten Oktave, punktuell ein mit Sprechgesang zu assoziierendes Ausdrucksmittel, wobei häufig Portamenti eingebaut werden (wie in Beispiel 4, Auftakt zu T. 2). Der Gesang hat erzählenden Charakter und erhält in Verbindung mit Bakers heller Stimmfarbe einen eigenen, fast kindhaft wirkenden Charme. Die Melodielinien sind insgesamt bewegt und anspruchsvoll gestaltet, und auch weitere Sprünge treten vermehrt auf. Ein Vibrato wird häufig bei den länger gehaltenen Tönen, hauptsächlich in der höheren Lage, verwendet. Jenes Element, das vom französischen Publikum besonders positiv aufgenommen wurde, war Bakers amerikanischer Akzent im Französischen: Er wurde zwar oft als fürchterlich bezeichnet, trug aber gerade deshalb zu ihrem Charme und Erfolg als Sängerin bei (vgl. BBC 2005).

Im ganzen Stück und besonders in der Strophe arbeitet Baker in ihrer Interpretation rhythmisch sehr differenziert: Die realisierte rhythmische Grundhaltung und Begleitung sind ternär; Baker singt die Melodie-Achtelnoten teilweise aber auch gerade, also binär (gekennzeichnet in der Transkription durch die eckigen Klammern, z.B. Beispiel 4, T.3), was eine abwechslungsreichere Melodiegestaltung und interessante Mischung mit der Begleitung ergibt. Teilweise wird die jeweils erste Achtelnote sogar stärker verlängert, was im Notenbild durch die Punktierung dargestellt wird (z.B. Beispiel 4, T.5). Zusätzlich singt sie einzelne Töne antizipiert (dargestellt durch den nach links weisenden Pfeil), andere jedoch retardiert (Pfeil nach rechts). Werden alle Töne eines ganzen Melodiebogens stark retardierend beziehungsweise antizipierend gesungen, wird dies mit einem langen Pfeil verdeutlicht: Dies wird beispielsweise in Takt 2 hörbar, wo alle markierten Töne unter dem Pfeil stark verspätet sind und als reizvoller Kontrast in Takt 3 binär realisierte Achtelnoten folgen.

Im zweiten Refrain B2 ergänzt Baker Lamys lang gehaltene Melodietöne mit Fill-Ins. Diese Einwürfe sind nicht improvisiert, sondern wurden laut Aussage von Bakers Zeitgenossen mitkomponiert und in der Partitur notiert (vgl. BBC 2005). Durch diesen Gesangspart tritt Bakers gesangliche Vorliebe und spezielle Stärke hervor, ihr koloraturartiger Sopran: Sie konnte in ihrem hohen Stimmregister mit einer besonderen Beweglichkeit und Leichtigkeit klare Linien, insbesondere sinnleere Silben-Linien, singen (siehe Beispiel 5). Diese spezifische Singart wurde zu einem ihrer Markenzeichen. Die eingefügten Phrasen in B2 sind einfach und bestehen aus wenigen, meist drei verschiedenen Tönen. Die ersten drei dieser Passagen singt Baker in binär realisierten Achtelnoten, wodurch sich ein Kontrast zu Lamys Melodie und der Begleitung ergibt. Der vierte Einwurf im Takt 7 ist jedoch von blues- 
artigem Charakter (zusätzliche Betonung der kleinen Septime ces ${ }^{2}$ von $\mathrm{Db}^{7}$ ), und auch die beiden nachfolgenden Einwürfe werden wieder ternär gesungen. In den letzten Takten des Refrains übernimmt Baker erneut die Melodie und endet schließlich mit einer Bewegung nach oben auf dem Schlusston $f^{2}$, mit Vibrato und einem ausklingenden decrescendo.
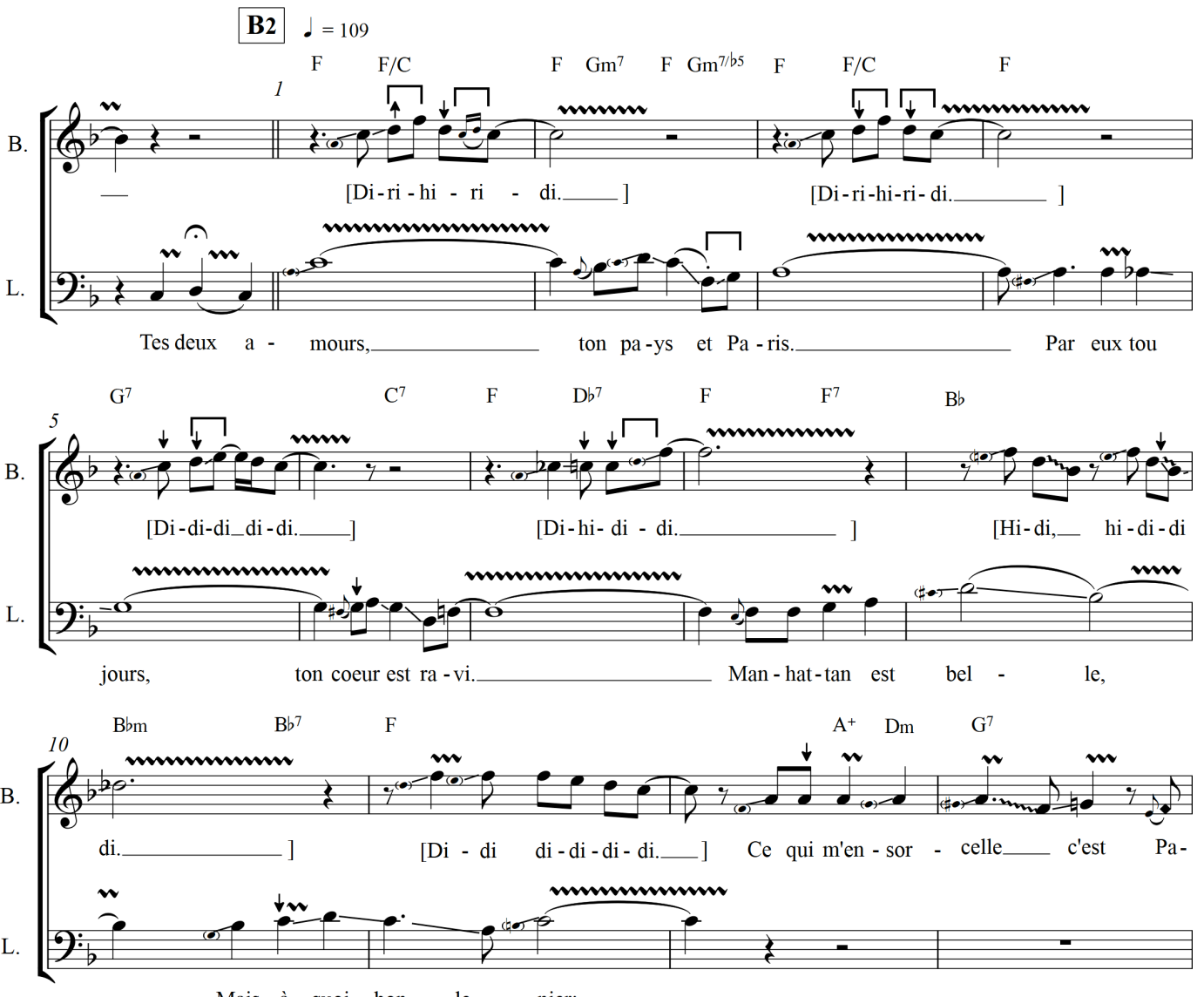

Mais à quoi bon le nier:

B.

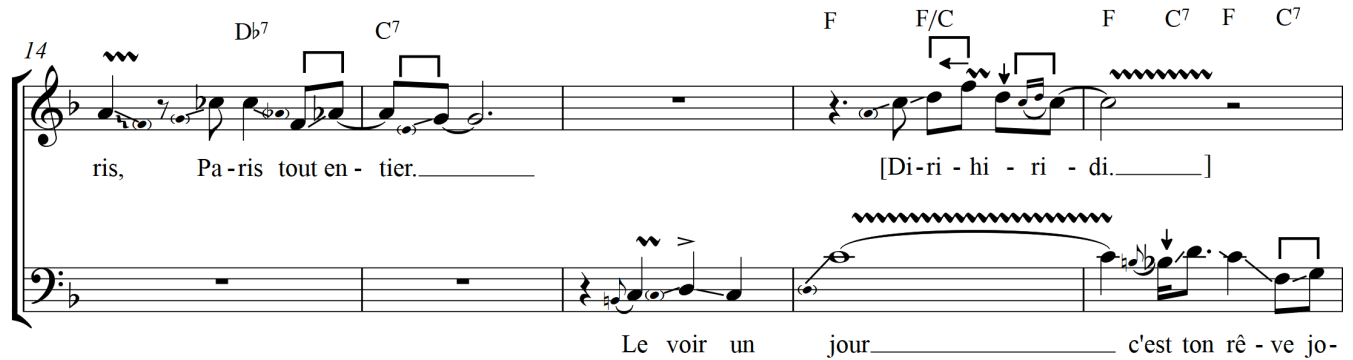

B

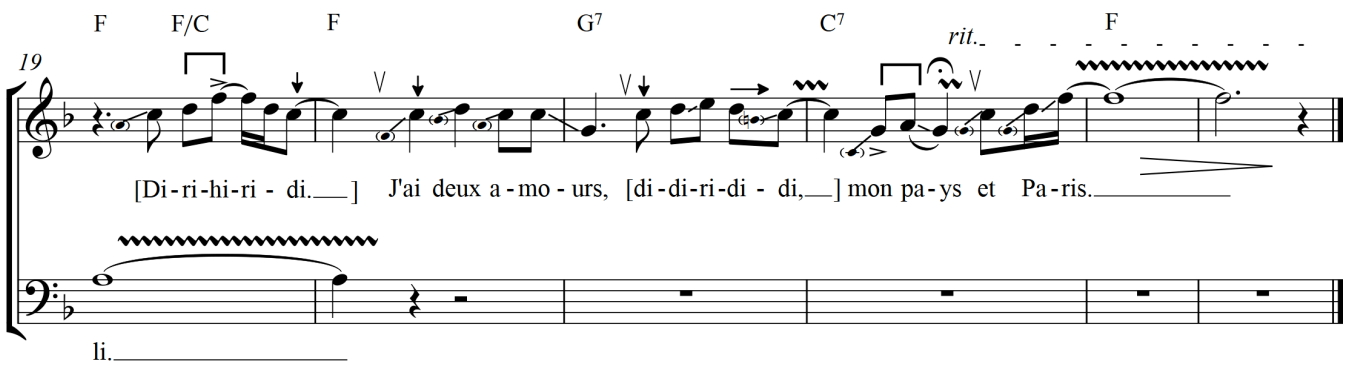

Beispiel 5: »J'ai deux amours«, B2 


\section{Das Baker-Image im Film}

Eine bemerkenswerte Visualisierung des Baker-Images enthält der 1934 produzierte Film Zouzou (Corona Films 1934). Nach der gescheiterten Hoffnung auf eine Liebesbeziehung mit ihrem Adoptivbruder Jean kehrt Zouzou, verkörpert von Baker, an jene Bühne zurück, auf der sie zu Ruhm gelangt ist. In einem überdimensionalen weißen Käfig sitzt sie kostümiert als weißer Vogel auf einer Schaukel und singt den Song »Haïti «. In einer Leichtigkeit wie losgelöst vom Körper entzieht sich ihre Stimme äußerer Einschreibungen und wird nur noch eine Hülle. Die junge Kreolin besingt ihren Herkunftsort und bedient auf ähnliche Weise wie in »J'ai deux amours« das Stereotyp der Heimatlosigkeit, der Sehnsucht nach der Heimat. Haiti ist für die Interpretin das Paradies, das schöne Land, wohin sie zurückkehren möchte, weil sie sich in der Ferne wie im Exil und in einem Käfig fühlt. Der Liedtext stammt von Géo Koger, die Musik von Vincent Scotto. Die Struktur ist eine dreiteilige Liedform A-B-A mit einem wiederkehrenden Refrain.

\section{»Haïti « ${ }^{7}$}

A (Refrain)

Ah! Qui me rendra mon pays,

Haïti.

C'est toi mon seul paradis, Haïti.

Ah! Dieu me rappelle

Tes forêts si belles,

Tes grands horizons

Loin de tes rivages.

La plus belle cage

N'est qu'une prison.

Oui! Mon désir, mon cri d'amour,

Haïti.

$\mathrm{C}^{\prime}$ est de te revenir un jour.

B (Strophe)

Oh, beau pays bleu, bien loin

Bien loin sous d'autres cieux.

Je vivais des jours heureux,

Mais tout est fini.

Seule dans mon exil aujourd'hui Je chante, le coeur meurtri:
Ach, wer wird mir mein Land zurückgeben,

Haïti.

Du bist mein einziges Paradies, Haiti.

Ach, wenn Gott mich erinnert

an deine so schönen Wälder,

die Weite deines Horizonts,

entfernt von deinen Küsten.

Der schönste Käfig

ist nur ein Gefängnis.

Ja, meine Sehnsucht, mein Schrei nach Liebe,

Haïti,

ist eines Tages zu dir zurückzukehren.

Oh, schönes blaues Land, wohl weit, wohl weit unter anderen Himmeln.

Ich erlebte glückliche Tage,

aber das ist Vergangenheit.

Allein in meinem Exil heute

singe ich mit schwerem Herzen:

7 Deutsche Übersetzung von den Autorinnen. 
In gesangstechnischer Hinsicht treten in Bakers Interpretation von »Haïti « ähnliche Charakteristika wie in »J'ai deux amours « auf: So hört man beispielsweise eine in melodischer und rhythmischer Hinsicht recht variabel gestaltete chansonartige Strophe, gemischt mit Elementen der Sprechstimme. Die höhere Lage des Refrains kostet Baker mit lang gehaltenen Tönen sowie Vibrato und diversen Umspielungen aus. Besonders im improvisatorisch gestalteten zweiten Refrain bewegt sie sich hauptsächlich in der zweiten Oktavlage und singt bis zum $c^{3}$ koloraturartige Linien, die - passend zu ihrer Inszenierung im Käfig - an einen Vogelgesang erinnern. In rhythmischer Hinsicht kann man wieder eine zum Teil fast übertrieben wirkende ternäre Realisierung neben einzelnen binär gesungenen Bewegungen hören.

Insgesamt wird in diesem Stück das sopranhafte Element in ihrer Stimme hervorgehoben: Dies wird besonders deutlich in der letzten Passage, einer Schlusskadenz, in der Baker - solistisch, wie in klassischer Tradition üblich - betont operettenhaft ihren hellen Koloratursopran präsentiert.

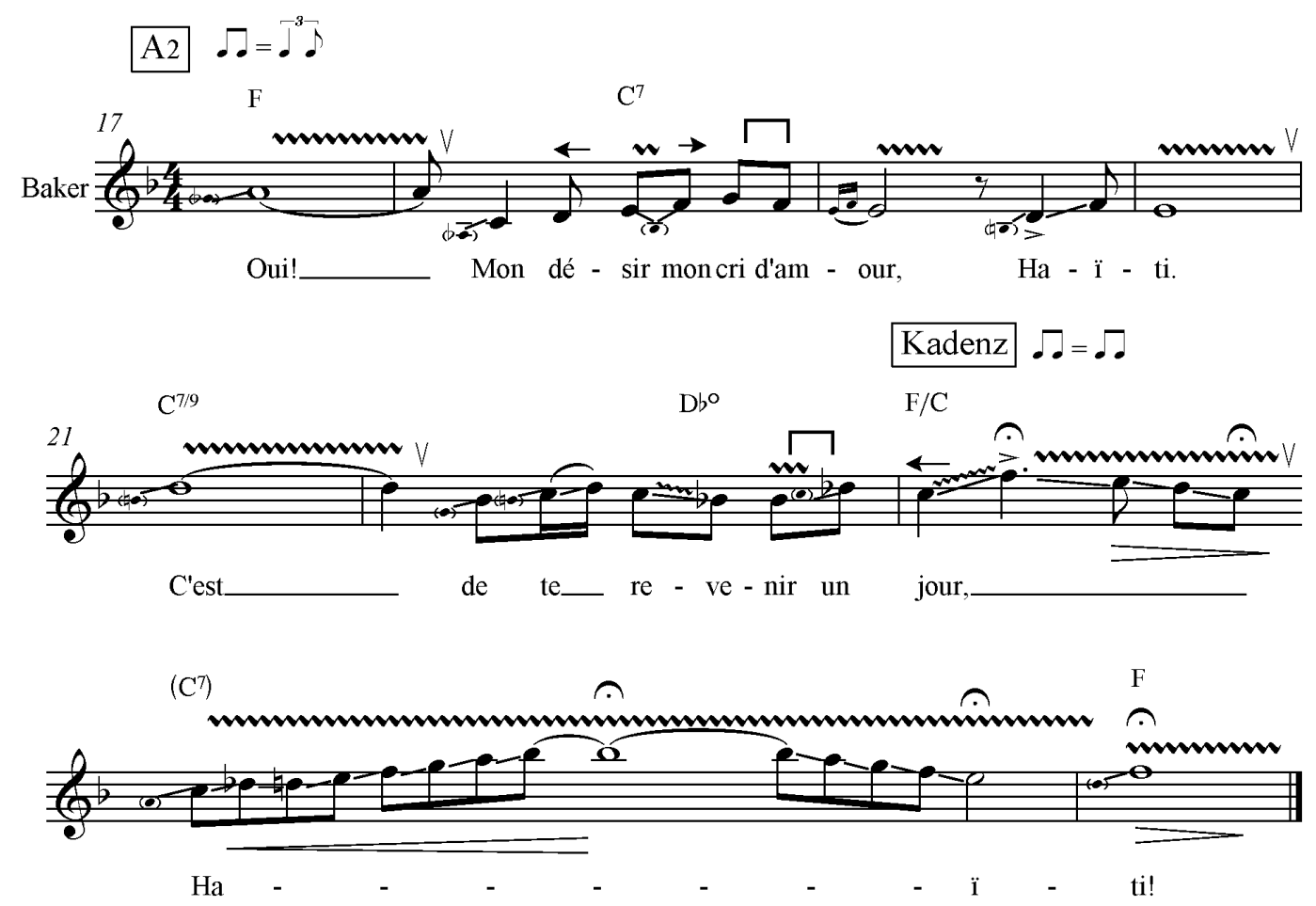

Beispiel 7: »Haïti«, Schlusskadenz

Wieder tun sich eine Reihe von Fragen bei der Betrachtung dieser Filmszene mit Zouzou im Käfig und der darin präsentierten Inszenierung Bakers auf: Ist der Vogel weiß oder schwarz? Symbolisiert er die Identifikation des weißen Betrachters mit dem begehrten Fremden, indem in der weißen Hülle das Eigene verschleiert durch Stereotypisierungen des Anderen verborgen liegt? Welch verhaltener Witz steckt hinter dieser artifiziellen Stimme, die sich in 
gesanglicher Hinsicht an die westliche Musiktradition, sich durch den Text des Liedes jedoch an den schwarzen Kontinent bindet und damit wiederum das Bild des Primitiven, sprich das Bananenrock-Image aufscheinen lässt?

\section{Fazit}

Die Figur der Josephine Baker gewinnt ihre besondere Faszination durch ihre Mehrdeutigkeit inmitten eines dialektischen Spannungsfeldes von Weiß und Schwarz. Entscheidend für die Entfaltung ihrer changierenden Facetten ist der Betrachter. Je nach eingenommener Perspektive erscheint sie als barbarisch-ursprünglich, als unzivilisiert und gleichzeitig als Inkarnation geheimer Wünsche, in der Art ihrer Bewegung als erotisch-geheimnisvoll und zugleich in ihren parodistischen Zügen als grotesk, aber auch gesellschaftskritisch, in ihrem Gesang als artifiziell, kindlich-naiv, aber auch als (begehrenswert) fremd. Baker inszeniert sich als Oberflächenphänomen, als Hülle, die mehr und mehr zur Hülle ihrer Betrachter wird. Damit werden diese zu den eigentlichen Performern.

\section{Literatur}

Borshuk, Michael (2001). »An Intelligence of the Body: Disruptive Parody through Dance in the Early Performances of Jospehine Baker. "In: Embodying Liberation. The Black Body in American Dance. Hg. v. Dorothea Fischer-Hornung und Alison D. Goeller. Hamburg: Lit Verlag, S. 41-58.

Butler, Judith (1990). Gender Trouble. Feminism and the Subversion of Identity. New York: Routledge.

Cheng, Anne Anlin (2011). Second Skin. Josephine Baker and the Modern Surface. Oxford: Oxford University Press.

Ehlers, Monika / Lezzi, Eva (Hg.) (2003). Fremdes Begehren: transkulturelle Beziehungen in Literatur, Kunst und Medien. Köln: Böhlau.

Gates, Henry Louis Jr (1984). »The Blackness of Blackness: A Critique of the Sign and the Signifying Monkey. «In: Black Literature and Literary Theory. Hg. v. dems. New York: Methuen, S. 285-322.

Gumbrecht, Hans Ulrich (2003). 1926. Ein Jahr am Rand der Zeit. Frankfurt/M.: Suhrkamp.

Herzhaft, Gérard (1992). Encyclopedia of the Blues. Fyetteville: The University of Arkansas Press.

Hölz, Karl (2002). Zigeuner, Wilde und Exoten: Fremdbilder in der französischen Literatur des 19. Jahrhunderts. Berlin: Schmidt.

Jules-Rosette, Bennetta (2007). Josephine Baker in Art and Life. The Icon and the Image. Champaign: University of Illinois Press.

Klein, Gabriele (2010). "Tanz als Aufführung des Sozialen. Zum Verhältnis von Gesellschaftsordnung und tänzerischer Praxis.« In: Konzepte der Tanzkultur. 
Wissen und Wege der Tanzforschung. Hg. v. Margrit Bischof und Claudia Rosiny. Bielefeld: Transcript.

Levine, Lawrence (1977). Black Culture and Black Consciousness: Afro-American Folk Thought from Slavery to Freedom. Oxford: Oxford University Press.

Salten, Felix (1924). »Wien, gib acht!«In: Neue Freie Presse, Wien, 10. Februar, S. 1-3; online unter: http://anno.onb.ac.at/cgi-content/anno?apm=0\&aid=nfp\& datum=19240210\&zoom=2 (Zugriff: 13.11.2011).

\title{
Diskographie
}

Baker, Josephine (1968). Encore! EMI Records SX 6264.

Baker, Josephine (1999). Complete Recorded Works 1926-1927. Document Records DOCD-5652.

\section{Filmographie}

BBC (2005). Josephine Baker: The First Black Superstar. Dokumentation, British Broadcasting Corporation; online unter: http://www.youtube.com/watch?v= Ggb_wGTvZoU (Zugriff: 13.11.2012).

Corona Films (1934). Zouzou; online unter: http://www.archive.org/details/Zouzo uAkaZouZoudecember211934 (Zugriff: 13.11.2012).

\begin{abstract}
In 1925 the young African-American dancer Josephine Baker went to Paris. After appearing in the show La Revue négre with a skirt made of bananas, she became a star practically overnight. Her self-presentation on stage and the implied staging of African and American culture fascinated not only the French audience, but attracted attention far beyond the borders of the country. Her performances were a stimulating mixture of art forms, with dance as the central element: she thrilled audiences with the Charleston and intrigued them with her exotic/erotic movements, in stark contrast to the classical dance tradition of Europe.

A closer look at Baker's performances at the height of her career, between 1925 and 1935, reveals multifaceted and increasingly sophisticated shadings. Having started as a dancer, she soon began singing as well: her vocal interpretations, well documented on recordings and in movies, complemented and completed her self-created images. By examining Baker's autobiographies, sound recordings and film footage, the artistic elements of her dancing and singing will be explored, highlighting the main facets of her performances and tracing the secret of her success.
\end{abstract}

